\title{
Dinuclear Complexes of Flexidentate Pyridine-Substituted Formazanate Ligands
}

\author{
Ge Mu and Thomas. S. Teets* \\ In this work we show the utility of flexidentate pyridyl-substituted formazanate ligands in assembling dinuclear coordination \\ complexes with iridium(III) and/or platinum(II) building blocks. The versatile binding modes of these ligands allow the \\ preparation of several different dinuclear structures, highlighting the potential of these formazanates to serve as redox- \\ active supporting ligands for multimetallic complexes. The dinuclear complexes are typically prepared in a stepwise strategy, \\ adding one metal unit at a time, with the coordination mode of the formazanate with the first metal dictating the binding \\ mode in the final dinuclear structure. Eight of the new complexes, including both mononuclear precursors and dinuclear \\ products, are structurally characterized by single-crystal X-ray diffraction, which along with NMR spectroscopy \\ unambiguously establish ligand binding modes and symmetries of the compounds. All complexes are characterized by UV- \\ Vis absorption spectroscopy and cyclic voltammetry. The frontier orbitals are localized on the formazanate ligand, and a \\ characteristic, intense formazanate-centered $\pi \rightarrow \pi^{*}$ absorption band is observed in the absorption spectrum. Structure- \\ property relationships are established, relating the ligand binding mode to the redox properties and spectroscopic features.
}

\section{Introduction}

Formazanates, the monoanionic form of formazans with a 1,2,4,5-tetraazapentadienyl (NNCNN) core, have become a well-known $\mathrm{N}$-chelating ligand class in recent years. They are structurally related to $\beta$-diketiminates, but the two additional nitrogen atoms in the backbone provide formazanates with more accessible redox properties and stronger visible absorption, due to their more stabilized LUMOs. $^{1-3}$ Formazanates have garnered considerable attention in coordination chemistry due to their ligand-based redox processes, which may facilitate multielectron redox transformations, ${ }^{4}$ bond activations ${ }^{5}$ and excited-state charge separation. ${ }^{6}$ A variety of formazanate complexes of many main group metals ${ }^{7-16}$ and first- and second-row transition metals ${ }^{17-}$ ${ }^{23}$ have been described, showcasing the versatile coordination chemistry of formazanate ligands and providing many examples of and much insight into the intense visible absorption and accessible redox states of formazanates. Some copper complexes can also mediate oxygen activation, ${ }^{24,25}$ certain cobalt and iron complexes exhibit unique magnetic characteristics, ${ }^{17,26}$ and boron complexes in many cases feature not only the tunable redox properties but also visible to nearinfrared photoluminescence, ${ }^{9-13}$ finding applications as cellimaging agents ${ }^{27,28}$ and electrochemiluminescence emitters. ${ }^{10}$ Our group has expanded the coordination chemistry of formazanates to third-row transition metals with a series of

\footnotetext{
a. University of Houston, Department of Chemistry, 3585 Cullen Blvd. Room 112, Houston, TX USA 77204-5003 Email: tteets@uh.edu

Electronic Supplementary Information (ESI) available: Experimental details, X-ray crystallographic tables, NMR spectra, and solvent-dependent absorption spectra. CCDC. For ESI and crystallographic data in CIF or other electronic format see DOI: $10.1039 / x 0 x x 00000 x$
}

heteroleptic cyclometalated platinum complexes and biscyclometalated iridium complexes, ${ }^{29-31}$ and accessed homoleptic azo-iminate platinum complexes and azo-1,2,3triazolide iridium complexes via hydrogenative cleavage or $[3+2]$ cyclization of formazanates, respectively. ${ }^{32,33}$

Compared to the numerous formazanate complexes where a single metal atom is chelated within the NNCNN framework, polynuclear compounds involving formazanates remain rare. Access to complex polynuclear formazanate complexes is desirable for a number of reasons; in addition to greatly expanding the fundamental coordination chemistry of formazanates, there is the possibility of designing compounds with enhanced or unusual redox, magnetic, or photophysical properties, enabled by electronic communication between the building blocks mediated by the redox-active formazanate. In addition, compared to the rigid polypyridyl-based bridging ligands that are ubiquitous in coordination chemistry, formazanates offer the advantage of greater coordination flexibility and redox potentials that can be more easily tuned over a wide range. There are a few existing categories of polynuclear complexes involving formazanates, with representative examples shown in Chart 1 . In some cases, the formazanate binds in a normal chelating mode, with other bridging moieties, either covalently attached to the formazanate periphery or added in as exogenous ligands, templating the polynuclear structure. This type of assembly was reported in two different works by the Gilroy group, one where boron difluoride formazanates are conjugated to platinum acetylide complexes, via aryl acetylene groups on the formazanate periphery, ${ }^{15}$ and another involving a cyclic trimeric boroxine compound, with the three boron atoms are linked by 


\section{Complexes with non-formazanate bridging ligands}

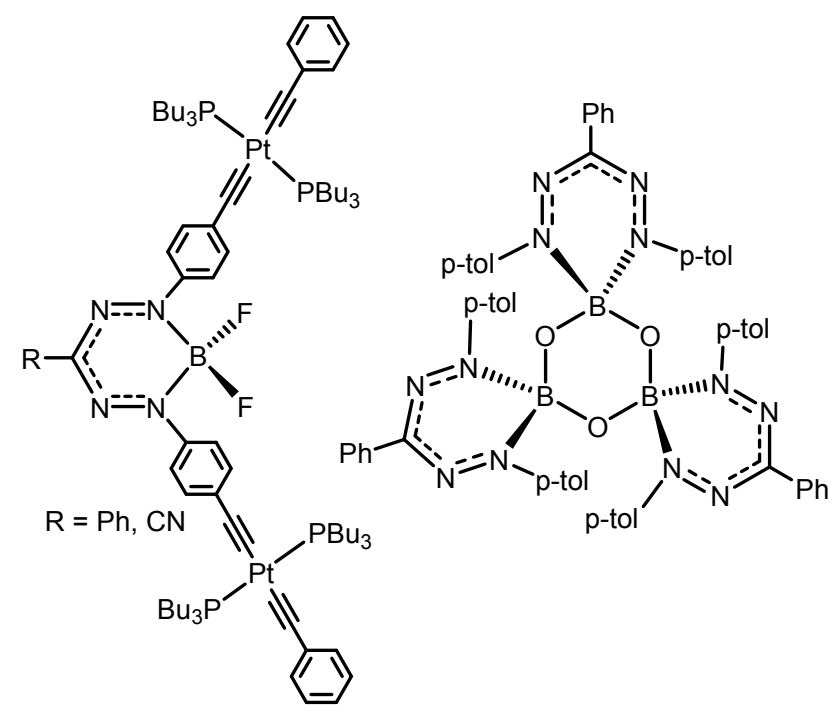

Complexes with formazanate bridging ligands

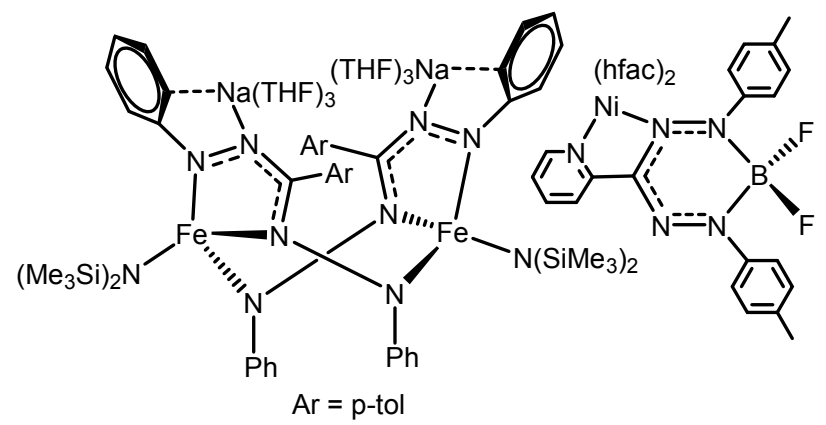

Chart 1. Representative polynuclear formazanate structures.

oxo bridges. ${ }^{34}$ In other cases, the formazanate itself is the polynucleating ligand and binds multiple metals at once. The Holland group isolated dimeric iron formazanate complexes which formed following alkali metal reduction of mononuclear iron formazanate precursors; the alkali metal cation templates the formation of the dimer, and the formazanate coordinates via a hybrid binding mode, chelating the iron through $\mathrm{N} 1$ and N4, with N5 bridging to the adjacent iron center. ${ }^{20}$ In another recent effort from the Gilroy group, formazanates substituted with a 2-pyridyl group were shown to be able to simultaneously bind $\mathrm{BF}_{2}{ }^{+}$and divalent nickel. ${ }^{12}$ With these few recent precedents in mind, we reasoned that the flexidentate pyridylsubstituted ligands introduced by Gilroy's group, and other related derivatives, could serve as versatile polynucleating ligands for a wide range of structures.

Having extensively studied the coordination chemistry of formazanates with $\operatorname{Ir}(\mathrm{III})^{31,33}$ and $\mathrm{Pt}(\mathrm{II}),{ }^{29,30}$ our initial effort presented herein includes a series of complexes containing different combinations of these two metals bridged by pyridylsubstituted formazanates. We elaborate the coordination chemistry of formazans with either 4- and 2-pyridyl substituents as the central aryl ring. The pyridyl substituents offer one additional coordination site, and we show in this work the diversity of coordination modes possible with these ligands. The dinuclear complexes that are presented in this study are either assembled in a stepwise fashion, adding one metal at a time to structure, or accessed via coordination-driven self-assembly. A total of 9 new formazanate complexes are introduced, with four mononuclear and four dinuclear complexes structurally characterized by single-crystal $X$-ray diffraction. All the compounds are interrogated by cyclic voltammetry and UV-Vis absorption spectroscopy, revealing the effects of the coordination mode and complex structure on the formazanatecentered frontier orbitals and visible absorption transitions.

\section{Results and Discussion}

\section{Synthesis and Structural Characterization}

In this work we prepared $9 \operatorname{Ir}(\mathrm{III}) / \mathrm{Pt}(\mathrm{II})$ pyridylformazanate complexes in total, which include mononuclear building blocks and dinuclear assemblies. We began our efforts with the new formazan 1,5-bis( $p$-tolyl)-3-(4-pyridyl)formazan (Fza); closely related derivatives with 1,5-diphenyl substitution have been described, ${ }^{35}$ but their coordination chemistry has only been described in one isolated report on homoleptic nickel bisformazanate complexes. ${ }^{36}$ Scheme 1 depicts the synthetic route of a mononuclear cyclometalated Ir(III) complex of Fza. Initially we used reaction conditions identical to those outlined in our previous report on cyclometalated iridium formazanate complexes, ${ }^{31}$ so our hypothesis was that Fza would chelate the iridium center through the formazanate backbone. We treated the chloro-bridged dimer $\left[\operatorname{Ir}\left(\mathrm{F}_{2} \mathrm{ppy}\right)_{2}(\mu-\mathrm{Cl})\right]_{2}$, where $\mathrm{F}_{2}$ ppy is 2(2,4-difluorophenyl)pyridine, with a stoichiometric amount of Fza in refluxing ethanol with excess triethylamine present. NMR spectroscopy confirmed isolation of a single product, but the ${ }^{1} \mathrm{H}$ NMR spectrum shows a downfield resonance at $15.79 \mathrm{ppm}$, consistent with the $\mathrm{N}-\mathrm{H}$ proton of the formazanate. We thus concluded that Fza binds to the $\operatorname{Ir}(\mathrm{III})$ center in a monodentate fashion through the nitrogen of the pyridyl substituent, with the

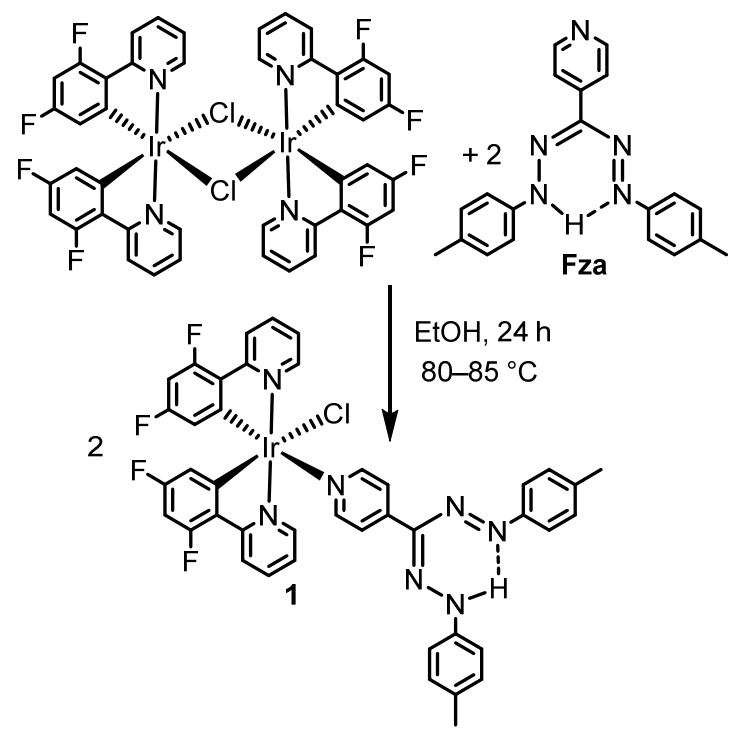

Scheme 1. Synthesis of complex 1. 


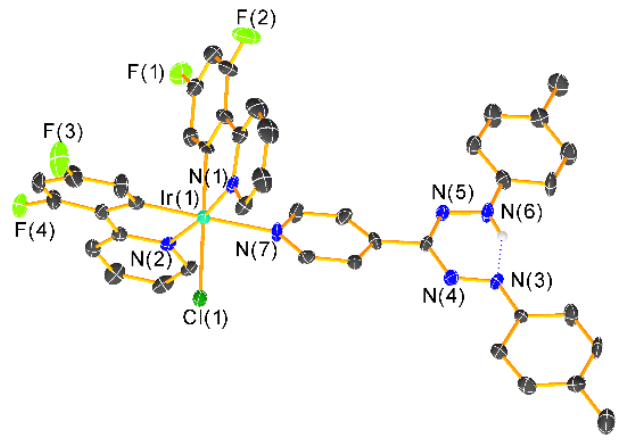

Figure 1. Molecular structure of complex 1, determined by single-crystal X-ray diffraction. Ellipsoids are shown at $50 \%$ probability level with solvent molecules and carbon-bound hydrogen atoms omitted.

chloride ligand still attached and an empty formazan backbone. The structure was unambiguously assigned by the crystal structure shown in Figure 1, which clearly shows the $\mathrm{K}^{1}$ coordination through pyridine. In addition, the $\mathrm{N}-\mathrm{H}$ hydrogen atom was located in the difference map, further confirming that the formazan core is unperturbed during this reaction. Recognizing that no deprotonation occurs during the formation of $\mathbf{1}$, as shown in Scheme 1 we removed triethylamine and performed the reaction without any base present, and the product was still formed in nearly identical isolated yield.

We then investigated complex $\mathbf{1}$ as a precursor for subsequent reactions targeting polynuclear complexes. Scheme 2 displays the synthetic route of the bimetallic cyclometalated Ir(III) complex 2, bridged by Fza. To install the second biscyclometalated iridium fragment, complex $\mathbf{1}$ was treated with a

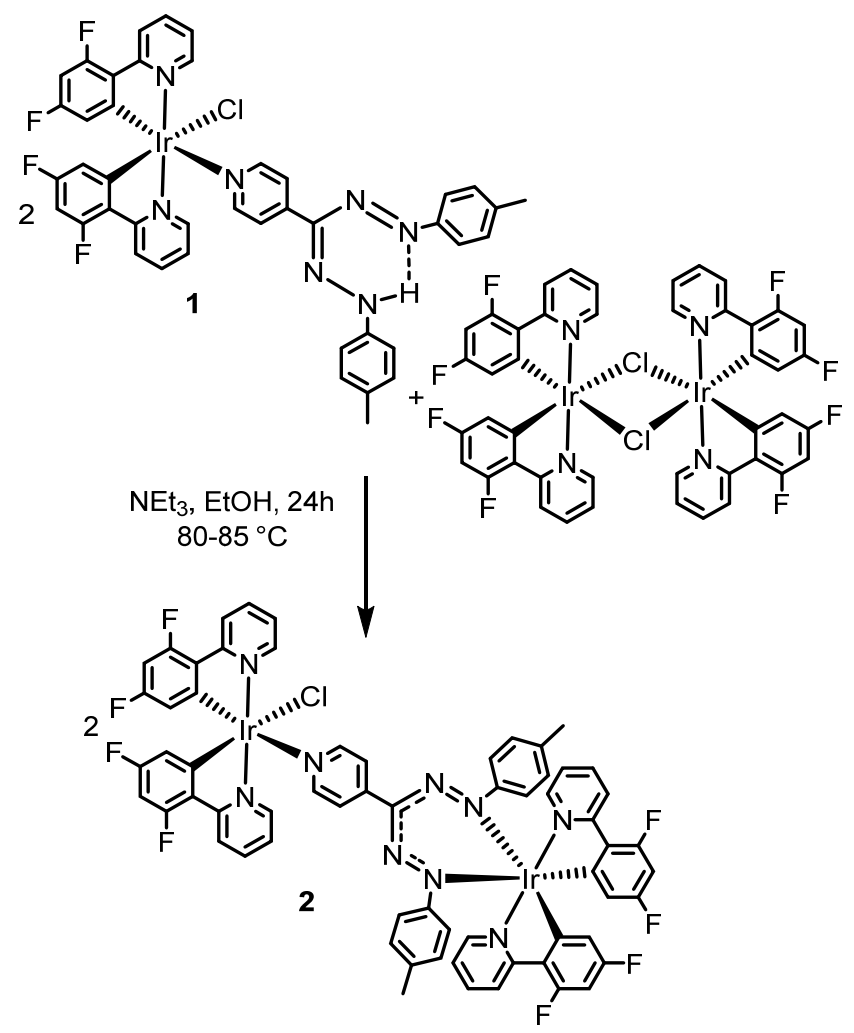

Scheme 1. Synthesis of complex 2.

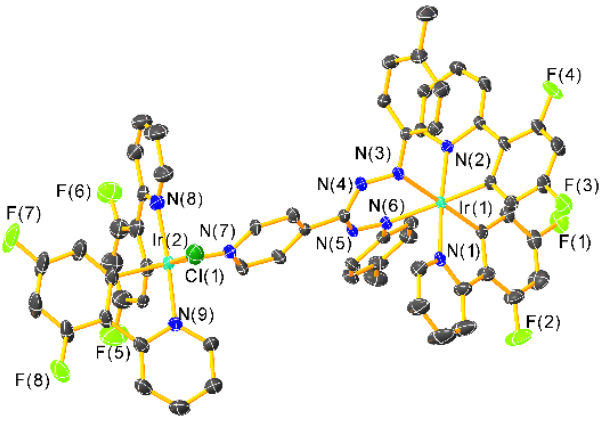

Figure 2. Molecular structure of complex 2, determined by single-crystal X-ray diffraction. Ellipsoids are shown at $50 \%$ probability level with solvent molecules and hydrogen atoms omitted.

stoichiometric amount of $\left[\operatorname{Ir}\left(\mathrm{F}_{2} \mathrm{ppy}\right)_{2}(\mu-\mathrm{Cl})\right]_{2}$ in refluxing ethanol with excess triethylamine. Following column chromatography, bimetallic complex 2 was isolated in moderate yield, 32\%. Formation of the bimetallic structure was evident from NMR spectroscopy. The characteristic downfield $\mathrm{N}-\mathrm{H}$ resonance, observed at $15.79 \mathrm{ppm}$ in 1, is no longer present. In addition, the ${ }^{1} \mathrm{H}$ and ${ }^{19} \mathrm{~F}$ NMR spectra display two sets of resonances for the $F_{2}$ ppy ligands; in one set there are two chemically equivalent $F_{2}$ ppy ligands, and in the other the two $F_{2}$ ppy ligands are chemically inequivalent. This observation suggests that one of the bis-cyclometalated iridium centers has local $C_{1}$ symmetry, as is the case in precursor complex $\mathbf{1}$, whereas the other has local $C_{2}$ symmetry. The structure of complex 2 thus includes the second $\left[\operatorname{Ir}\left(\mathrm{F}_{2} \mathrm{ppy}\right)_{2}\right]^{+}$fragment being chelated by $\mathrm{N} 1$ and $\mathrm{N} 5$ of the formazanate core, which is often termed the "closed" form of the formazanate. ${ }^{31}$ This binding mode was confirmed by $\mathrm{X}$ ray crystallography, with the structure shown in Figure 2 . The flexidentate ligand Fza exhibits a dual binding mode in the structure of $\mathbf{2}$, with the formazanate core chelating one metal center and the 4-pyridyl ring bound to the other. This confirms that the overall outcome of the reaction shown in Scheme 2 is to use complex $\mathbf{1}$ as a "metalloligand" to chelate a second iridium fragment.

The same binding mode of Fza observed in complex $\mathbf{2}$ was also observed with cyclometalated platinum. Whereas no reaction is observed between Fza and $\mathrm{Pt}\left(\mathrm{N}^{\wedge} N\right) \mathrm{Cl}_{2}$ precursors $\left(\left(\mathrm{N}^{\wedge} \mathrm{N}\right.\right.$ is $2,2^{\prime}$-bipyridine (bpy) or 4,4'-di-tert-butyl-2,2'-bipyridine (tbbpy)) in the presence of base, Fza does react with the chlorobridged dimer $[\mathrm{Pt}(\mathrm{ppy})(\mu-\mathrm{Cl})]_{2}$, as shown in Scheme 3. A 1:2 reaction between $[\mathrm{Pt}(\mathrm{ppy})(\mu-\mathrm{Cl})]_{2}$ and $\mathrm{Fza}$ in refluxing ethanol with excess triethylamine produces two products, a bimetallic complex 3 (major product) and chelated monoplatinum complex 4 (minor product). Crude NMR spectra indicate a ca. 3:1 ratio of the two products, and upon chromatographic separation complex 3 is obtained in 64\% yield (based on the platinum precursor), whereas 4 is obtained in $12 \%$ yield. The formation of both products during the same reaction suggests that monoplatinum complex $\mathbf{4}$ may be an intermediate in the formation of diplatinum complex $\mathbf{3}$. In addition, comparing the reaction with cyclometalated platinum shown in Scheme 3 to the reaction with iridium in Scheme 2 shows that Fza more readily chelates $\mathrm{Pt}(\mathrm{II})$ than $\mathrm{Ir}(\mathrm{III})$ and does not form products with platinum that are exclusively bound through the pyridine. 

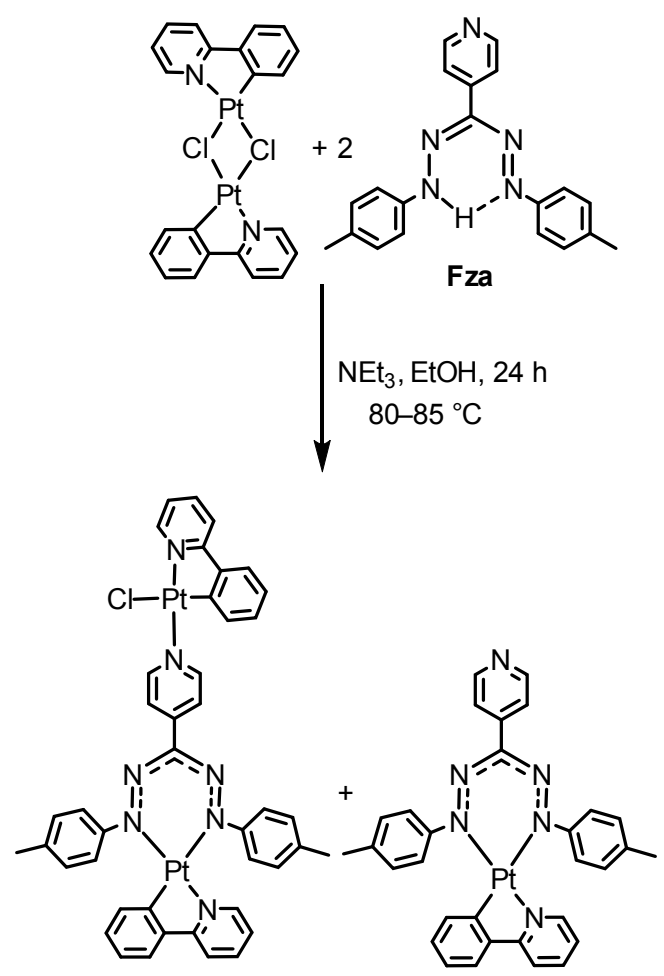

4

Ratio

3

Scheme 3. Synthesis of complexes $\mathbf{3}$ and $\mathbf{4}$

The structure of complex $\mathbf{4}$ was verified by X-ray crystallography (Figure 3), and is very similar to several other structurally characterized $\operatorname{Pt}\left(\mathrm{C}^{\wedge} \mathrm{N}\right)$ (formazanate) complexes from our group. ${ }^{29,30}$ We were unable to obtain single crystals of diplatinum complex 3, but NMR spectroscopy and mass spectrometry are all consistent with the proposed structure. In particular, the ${ }^{1} \mathrm{H}$ NMR spectrum of 3 shows two sets of resonances for the ppy ligands, indicating two chemically inequivalent $[\mathrm{Pt}(\mathrm{ppy})]^{+}$fragments, and one set of resonances attributed to Fza. All of these observations are consistent with the same binding mode for Fza in diplatinum complex $\mathbf{3}$ as is observed in diiridium complex $\mathbf{2}$ (Scheme 2 and Figure 2).

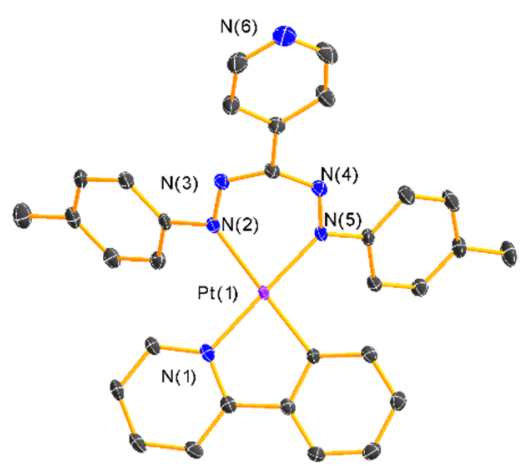

Figure 3. Molecular structure of complex 4, determined by single-crystal X-ray diffraction. Ellipsoids are shown at $50 \%$ probability level with hydrogen atoms omitted.
We also found that 4-pyridyl-substituted Fza can support a heterobimetallic complex, in this case prepared by a one-pot self-assembly. Recognizing that Fza has two different preferred binding modes with iridium and platinum (see Schemes 1 and 3) we treated equimolar amounts of $\left[\operatorname{Ir}\left(\mathrm{F}_{2} \mathrm{ppy}\right)_{2}(\mu-\mathrm{Cl})\right]_{2}$ and $[\mathrm{Pt}(\mathrm{ppy})(\mu-\mathrm{Cl})]_{2}$ with two equivalents of $\mathbf{F z a}$, in the presence of triethylamine in refluxing ethanol (Scheme 4). Clean selfassembly of the heterobimetallic complex $\mathbf{5}$ was observed, and following recrystallization the product was isolated in good yield (72\%). ${ }^{19} \mathrm{~F}$ NMR spectroscopy evinces local $C_{1}$ symmetry at the iridium center, with four distinct resonances for the $F_{2} p p y$ fluorine atoms, and the ${ }^{1} \mathrm{H}$ NMR spectrum, while complex in the aromatic region, shows the expected number of peaks and integration values for the proposed structure. Single crystals of complex $\mathbf{5}$ were obtained, with the structure determined by $\mathrm{X}$ ray diffraction depicted in Figure 4. The binding mode of Fza is clearly confirmed in the structure, with chelation to the cyclometalated platinum fragment and $\mathrm{K}^{1}$ binding through pyridine at the iridium site. As observed in complex $\mathbf{4}$ and many other platinum formazanate complexes from our group, ${ }^{29,30}$ the formazanate in complex $\mathbf{5}$ binds to the platinum in a "dragonfly" conformation, where the backbone carbon and 4-pyridyl substituent in the formazanate are splayed out of the platinum coordination plane. Looking at select bond distances, we note that the $\mathrm{Ir}-\mathrm{N}$ (pyridyl) bond distance in 5, 2.165(8) $\AA$, is very similar to, but slightly shorter than, the analogous distance in complex 1, which averages 2.184(11) ^ for two crystallographically independent molecules. This observation suggests that the electronic nature of the pyridine is minimally

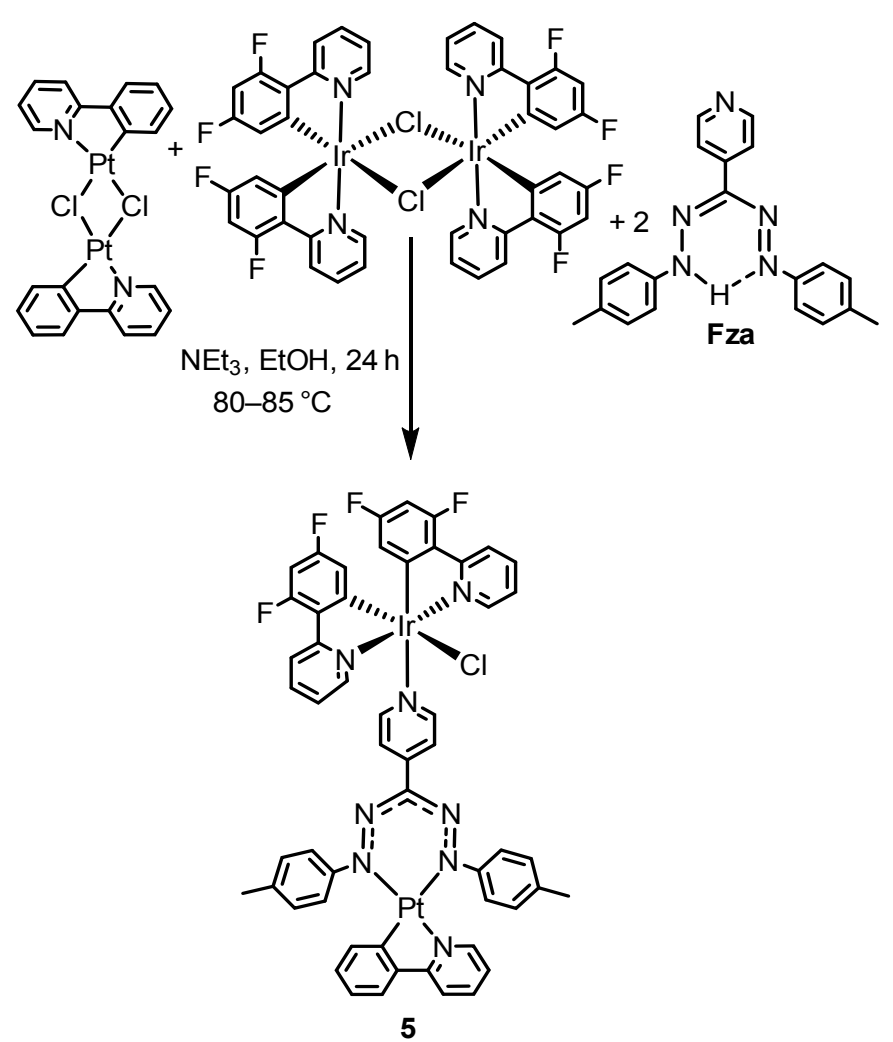

Scheme 4. Synthesis of complex 5. 


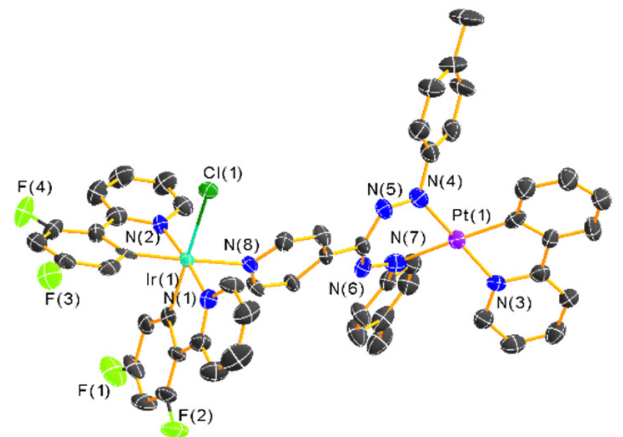

Figure 4. Molecular structure of complex $\mathbf{5}$, determined by single-crystal X-ray diffraction. Ellipsoids are shown at $50 \%$ probability level with solvent molecules and hydrogen atoms omitted.

altered when Fza coordinates to a metal. Similarly, the formazanate Pt-N distances, 2.037(2) $\AA$ and 2.088(2) $\AA$ in monoplatinum complex 4 , are only slightly shifted in bimetallic complex 5, where the same distances are 2.000(9) $\AA$ and 2.107(10) A.

The 2-pyridyl isomer Fzb does not react cleanly with cyclometalated iridium precursors, but we were able to construct diplatinum complexes with this ligand, exhibiting two distinct binding modes. Two monoplatinum precursor compounds $\mathbf{6}$ and $\mathbf{7}$ with a chelated Fzb were prepared as described in Scheme 5, and their structures were confirmed by NMR spectroscopy and single-crystal $\mathrm{X}$-ray diffraction (Figure 5). Of note, complex 6 represents a new structure type in platinum formazanate chemistry, with the neutral tbbpy and monoanionic Fzb supporting a cationic Pt(II) complex with an outer-sphere chloride anion.

We reasoned that complexes $\mathbf{6}$ and $\mathbf{7}$ could serve as chelating metalloligands, using the 2-pyridyl and an adjacent nitrogen atom in the formazanate core to chelate the second
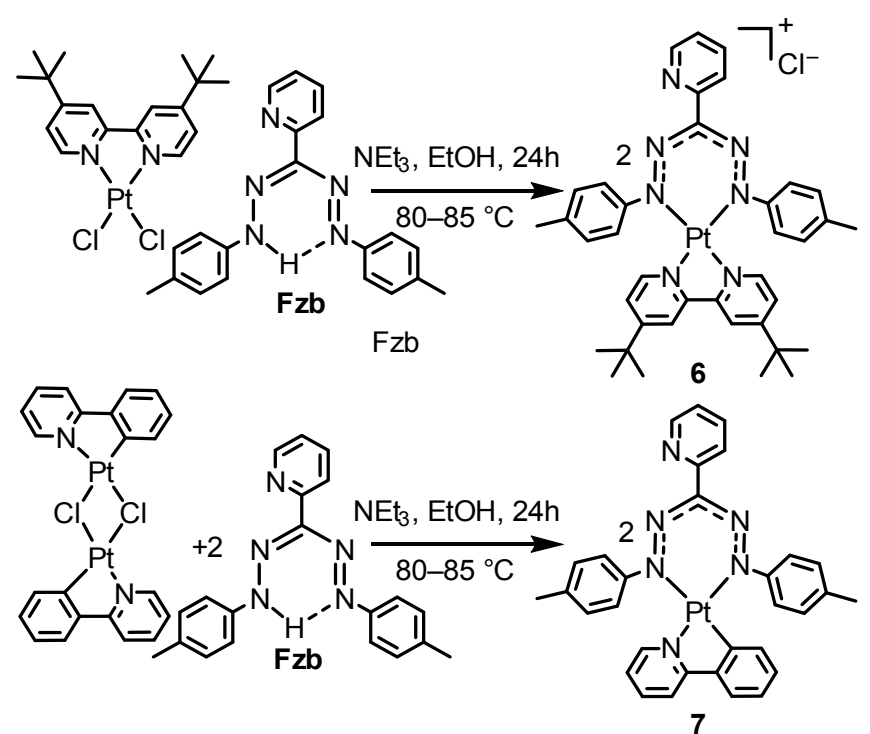

Scheme 5. Synthesis of complexes 6 and 7 .

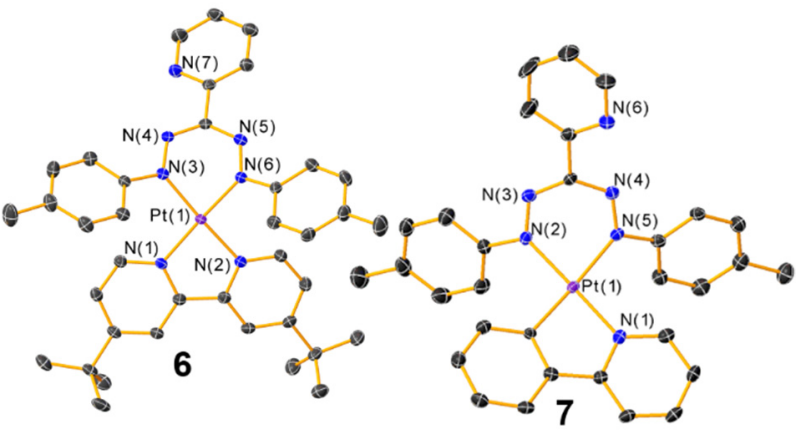

Figure 5. Molecular structure of complexes $\mathbf{6}$ and 7, determined by single-crystal X-ray diffraction. Ellipsoids are shown at $50 \%$ probability level with the chloride counterion (complex 7), solvent molecules, and hydrogen atoms omitted.

metal, in the same manner observed by Gilroy's group previously (see Chart 1 ). ${ }^{12}$ We first introduced the labile platinum precursor $\mathrm{Pt}(\mathrm{DMSO}){ }_{2} \mathrm{Cl}_{2}$ to complex 6 , anticipating the formation of a complex with $\mathrm{PtCl}_{2}$ chelated by the 2-pyridyl and an adjacent formazanate nitrogen, as shown in Scheme 6. The ${ }^{1} \mathrm{H}$ NMR spectrum of the resulting product was consistent with the formation of a more rigid product with the pyridine locked into a single conformation by virtue of coordination. Specifically, the formazanate $\mathrm{CH}_{3}$ resonances, which are chemically equivalent in $\mathbf{6}$ by virtue of free rotation of the 2pyridyl ring, split substantially in the product, separated by 0.2 ppm. In addition, the tert-butyl ${ }^{1} \mathrm{H}$ resonances likewise occur at different chemical shifts, albeit minimally split. However, the crystal structure of complex 8, displayed in Figure 6, reveals that a rearrangement occurs during the reaction, whereby the chloride counterion opens up the chelated formazanate at the $[\mathrm{Pt}(\mathrm{tbbpy})]^{2+}$ site, and the formazanate splays open to bind the $\mathrm{PtCl}_{2}$ through the 2-pyridyl moiety and N1 of the formazanate

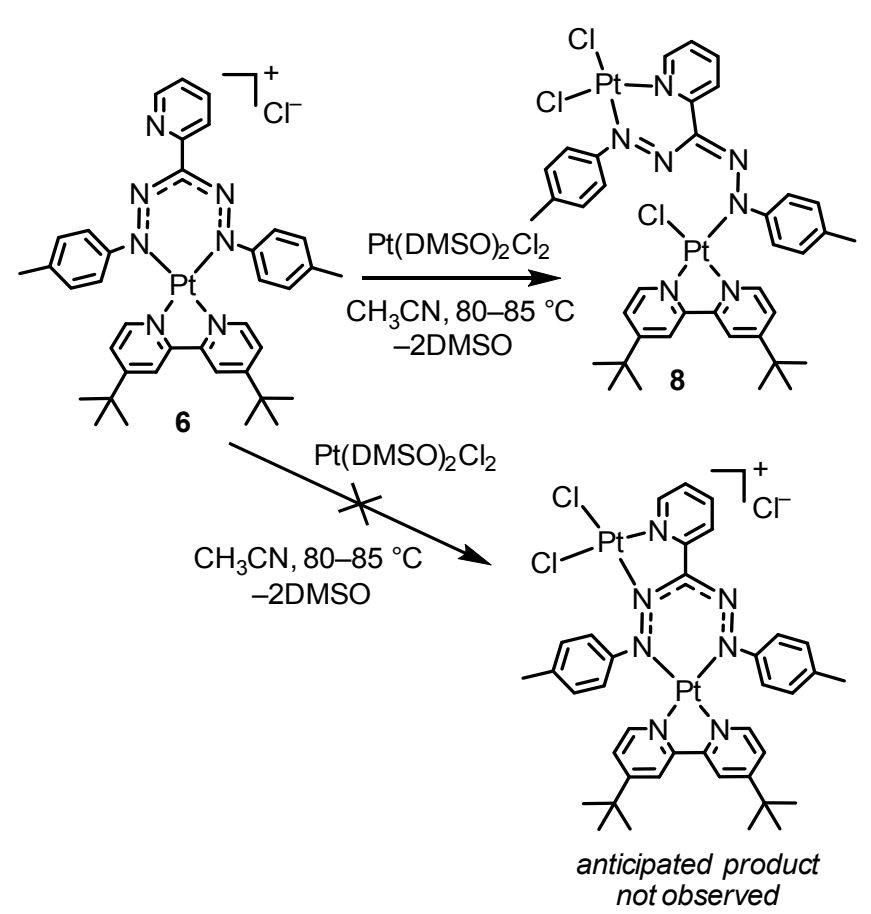

Scheme 6. Synthesis of complex 8 . 


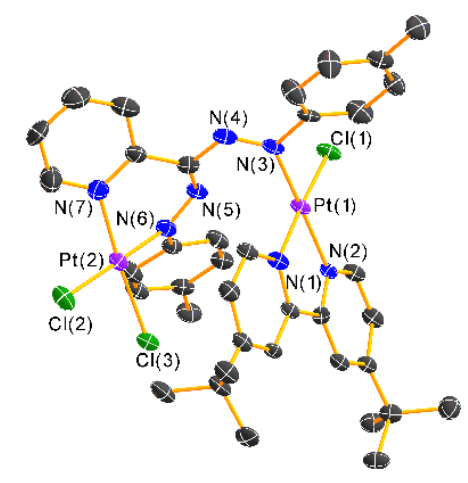

Figure 6. Molecular structure of complex $\mathbf{8}$, determined by single-crystal X-ray diffraction. Ellipsoids are shown at $50 \%$ probability level with solvent molecules and hydrogen atoms omitted.

core, forming a 6-membered chelate ring. The structure of $\mathbf{8}$ represents a new and unexpected binding mode for the flexidentate formazan Fzb, but nonetheless does show that this ligand can template dinuclear transition metal complexes.

Applying the same reaction conditions with neutral cyclometalated complex 7, we presumed that binding of $\mathrm{PtCl}_{2}$ would also occur, possibly via the same binding mode as in complex $\mathbf{8}$, but likely avoiding opening of the platinumformazanate since there is not an additional equivalent of $\mathrm{Cl}^{-}$ present in the reaction. Treating complex 7 with $\mathrm{Pt}(\mathrm{DMSO}){ }_{2} \mathrm{Cl}_{2}$ resulted in the formation of a new product, but the ${ }^{1} \mathrm{H} N M R$ spectrum did not match the expected product that would form from binding $\mathrm{PtCl}_{2}$. In particular, the ${ }^{1} \mathrm{H}$ NMR spectrum of the product has fewer aromatic resonances than the starting material (7), and one resonance occurs very far downfield, at

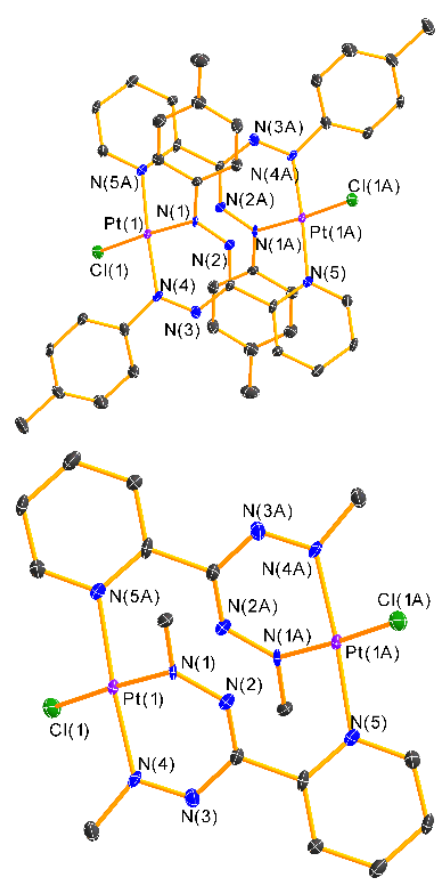

Figure 7. Molecular structure of complex $\mathbf{9}$, determined by single-crystal X-ray diffraction. Ellipsoids are shown at $50 \%$ probability level with solvent molecules and hydrogen atoms omitted. The bottom structure more clearly shows the coordination environment and bimetallic core, eliminating the $p$-tolyl substituents except for the carbon atoms bound to the formazanate nitrogen atoms.

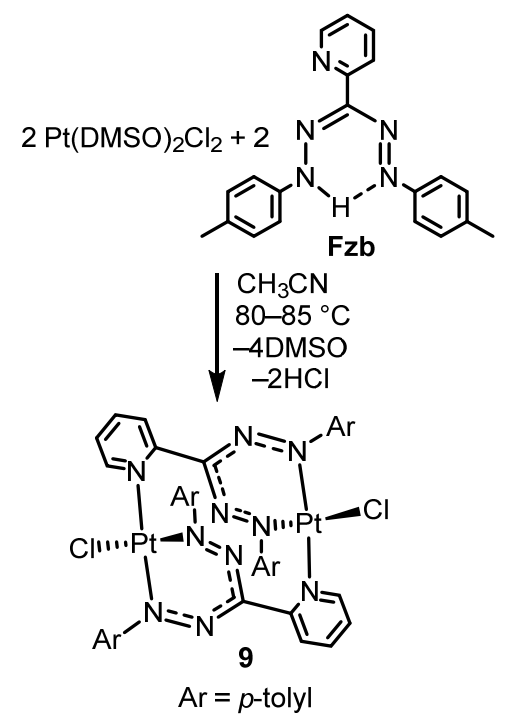

Scheme 7. The synthesis of complex 9.

$13.70 \mathrm{ppm}$. We determined by single-crystal X-ray diffraction that the product that formed (Figure 7) is a $C_{2}$-symmetric neutral diplatinum formazanate complex of the formula $\mathrm{Pt}_{2}(\mathbf{F z b}){ }_{2} \mathrm{Cl}_{2}$ (9), where each Fzb ligand chelates one platinum center and bridges to the other with the 2-pyridyl group. Thus the coordination geometry of each platinum includes the two nitrogen atoms from a chelating formazanate, a pyridyl nitrogen from the neighboring formazanate, and a terminal chloride. The fate of the $[\mathrm{Pt}(\mathrm{ppy})]^{+}$fragment that is lost during this reaction is not known, but we did find, as depicted in Scheme 7, that product 9 could be prepared rationally and much more efficiently by simply treating $\mathrm{Pt}(\mathrm{DMSO}){ }_{2} \mathrm{Cl}_{2}$ with equimolar Fzb in refluxing acetonitrile, allowing the diplatinum complex to be isolated in $64 \%$ yield. Examining the structure in Figure 7, we note that the platinum centers are separated by a large distance, $4.78 \AA$, indicating they are non-interacting, and we presume that the very downfield chemical shift in the ${ }^{1} \mathrm{H}$ NMR spectrum $(13.70 \mathrm{ppm})$ is attributed to the protons at the 6positions of the 2-pyridyl rings, which are in a locked comformation that result in close approach (2.53 $\AA$ ) to $\mathrm{N}(3)$ and $N(3 A)$.

\section{UV-Vis absorption spectroscopy}

Recognizing that formazanates are well-known for their redox and optical properties, this study provides an opportunity to assess how the binding mode of flexidentate formazanates influences the frontier orbitals and excited states. The absorption spectra and the corresponding visual color of the complexes depend strongly on the binding mode. UV-Vis spectra for the complexes are shown in Figure 8, with a summary of the data in Table 1 . The plots in Figure 8 are arranged by the nuclearity of the respective complex. Figure $8 \mathrm{a}$ shows the four mononuclear complexes $(\mathbf{1}, \mathbf{4}, \mathbf{6}$, and 7) with their bimetallic derivatives $(\mathbf{2}, \mathbf{3}, \mathbf{5}, \mathbf{8}$, and 9) shown directly below in Figure $8 \mathrm{~b}$, and the spectra of the free formazans are included in Figure 8c. 

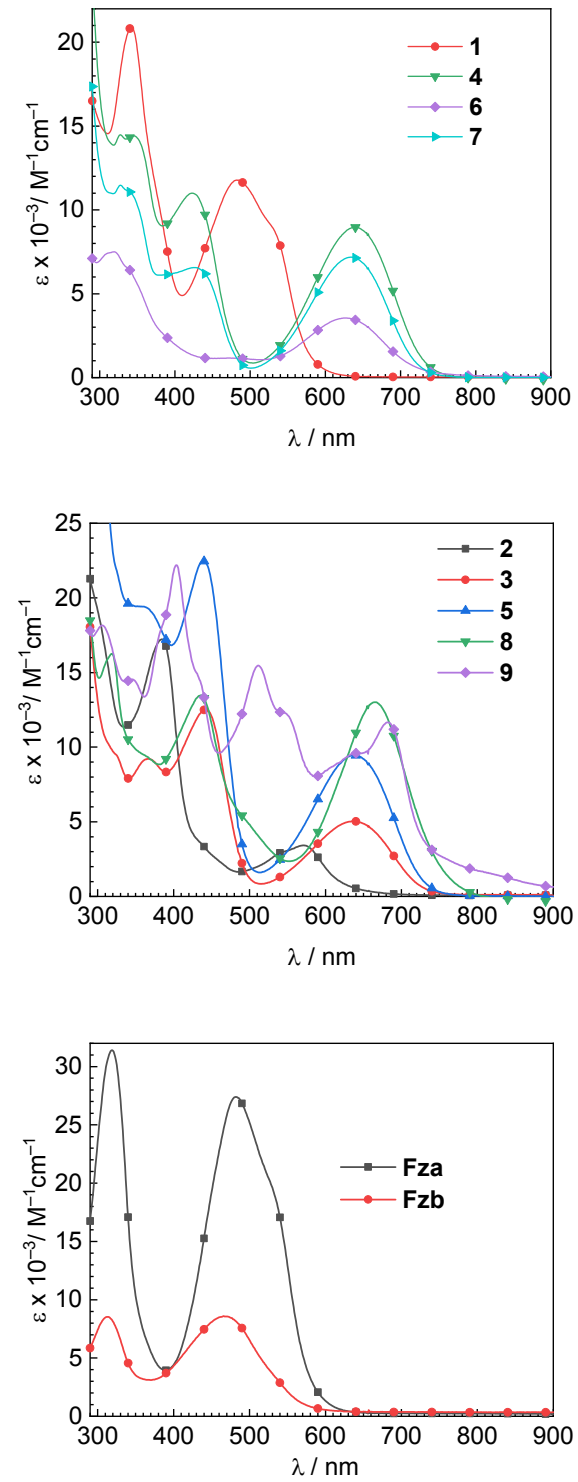

Figure 8. Overlaid electronic absorption spectra of (a) mononuclear complexes, (b) bimetallic complexes, and (c) free formazans Fza and Fzb. Spectra were recorded in tetrahydrofuran (THF) solution at room temperature. Data was collected in intervals of $1 \mathrm{~nm}$, and symbols are included on each plot are to help distinguish the overlaid spectra.

Apart from intense peaks in the UV $(\lambda<350 \mathrm{~nm})$ attributed to localized $\pi \rightarrow \pi^{*}$ transitions of the aryl substituents from both $\mathrm{C}^{\wedge} \mathrm{N}$ and formazanate, a notable absorption between $460 \mathrm{~nm}$ and $700 \mathrm{~nm}$ stands out among all compounds, which is assigned as a $\pi \rightarrow \pi^{*}$ within the formazanate framework. The free formazans have similar UV-vis absorption wavelengths, with the low-energy transition occurring $482 \mathrm{~nm}$ in Fza and $466 \mathrm{~nm}$ in Fzb. Coordination through the 4-pyridyl substituent of Fza, as observed in complex 1, has a minimal effect on the absorption wavelength, which occurs at nearly identical wavelength (483 $\mathrm{nm}$ ) in this complex. When the formazanate chelates the metal center, as observed in the other complexes described here, we observe a sizeable red-shift of the $\pi \rightarrow \pi^{*}$ transition, as we have previously noted in studying many other $\mathrm{Pt}$ and $\mathrm{Ir}$ formazanates. ${ }^{29-31}$ The magnitude of this shift depends on the metal that is chelated; in dinuclear complex $\mathbf{2}$, the only member of the series where the formazanate chelates to iridium, the low-energy absorption shifts to $571 \mathrm{~nm}$. In the Fza complexes where $\mathrm{Pt}$ is chelated (3-5), the corresponding absorption maxima span $635-640 \mathrm{~nm}$. Comparison of the mononuclear complex Pt(ppy)(Fza) (4) $\left(\lambda_{\max }=640 \mathrm{~nm}\right)$ to dinuclear complexes 3 and 5, where the 4-pyridyl is coordinated to a secondary metal center, again shows minimal impact of 4-pyridyl coordination on the absorption wavelength.

Table 1. Summary of UV-Vis absorption data recorded in tetrahydrofuran (THF) at room temperature.

\begin{tabular}{cc}
\hline & $\lambda / \mathrm{nm}\left(\varepsilon \times 10^{-3} / \mathrm{M}^{-1} \mathrm{~cm}^{-3}\right)$ \\
\hline Fza & $319(31), 482(27), 535(\mathrm{sh})(19)$ \\
Fzb & $313(8.5), 466(8.6)$ \\
$\mathbf{1}$ & $343(21), 483(12), 530(\mathrm{sh})(8.9)$ \\
$\mathbf{2}$ & $384(17), 571(3.4)$ \\
$\mathbf{3}$ & $325(\mathrm{sh})(9.4), 367(9.2), 442(13), 635(5.0)$ \\
$\mathbf{4}$ & $328(14), 346(14), 424(11), 640(9.0)$ \\
$\mathbf{5}$ & $364(\mathrm{sh})(19), 440(22), 636(9.5)$ \\
$\mathbf{6}$ & $320(7.5), 336(\mathrm{sh})(6.6), 626(3.6)$ \\
$\mathbf{7}$ & $328(11), 341(\mathrm{sh})(11), 428(6.6), 634(7.2)$ \\
$\mathbf{8}$ & $319(16), 436(13), 664(13)$ \\
$\mathbf{9}$ & $306(18), 349(\mathrm{sh})(14), 384(\mathrm{sh})(18), 404(22), 430(\mathrm{sh})(15)$, \\
& $511(15), 548(\mathrm{sh})(12), 649(\mathrm{sh})(9.6), 682(12)$ \\
\hline
\end{tabular}

In the Fzb series, mononuclear precursors 6 and $\mathbf{7}$ behave similarly to the other Pt chelates. The $\pi \rightarrow \pi^{*}$ transition in Fzb occurs at $466 \mathrm{~nm}$, and shifts to $626 \mathrm{~nm}$ in [Pt(tbbpy)(Fzb)](Cl) (6) and $634 \mathrm{~nm}$ in Pt(ppy)(Fzb) (7), indicating a small effect of the secondary ligand on the absorption energy. When complex 6 binds an equivalent of $\mathrm{PtCl}_{2}$ to form complex $\mathbf{8}$, which involves a rearrangement of the formazanate binding mode (see Scheme 6), the low-energy absorption band shifts significantly from 626 $\mathrm{nm}$ to $664 \mathrm{~nm}$, indicating that the $\pi \rightarrow \pi^{*}$ gap is sensitive to the ligand conformation and/or to the coordination of a second metal ion to the formazanate. Finally, diplatinum complex 9 has the most distinct UV-vis absorption spectrum in the series. The lowest-energy maximum in $\mathbf{9}$ occurs at the longest wavelength in the series, $682 \mathrm{~nm}$, and involves a high-energy shoulder at $649 \mathrm{~nm}$. Moreover, complex 9 has a second set of overlapped, intense visible bands, at 511 and $548 \mathrm{~nm}$, in a region of the spectrum where the other complexes are transparent. The origin of this second visible band is not immediately obvious from the available data, but we propose the most likely explanation for the appearance of the absorption spectrum in 9 is that each formazanate has a $\pi \rightarrow \pi^{*}$ excited state, and the two states mix through configuration interaction to produce two states that give rise to the two intense visible absorption bands.

UV-Vis spectra were also recorded in three solvents of varying polarity (toluene, THF, and $\mathrm{MeOH}$ ), shown in Figure S24, with a summary of data in Table S3. The low-energy absorption bands of most complexes exhibit small but measureable solvatochromism, indicating some amount of charge-transfer character in the excited state. For complexes with a chelated formazanate the solvatochromism is typically larger for $\mathrm{Pt}$ complexes vs. Ir, in line with what we have previously reported. Electrochemistry 
The redox properties of all complexes were evaluated by cyclic voltammetry, to determine the effect of formazanate binding mode on the redox potentials and frontier orbitals. Overlaid voltammograms shown in Figure 9 and redox potentials are summarized in Table 2. Each complex shows one oxidation and one reduction feature within the accessible electrochemical window. We note that complex 1, which contains a neutral formazan coordinated to iridium through the pyridine, is the most difficult to oxidize in the series, whereas in the rest of the compounds, with formally monoanionic metalbound formazanate cores the oxidation waves are cathodically shifted. In most cases other than complexes 8 and 9, the reduction waves are electrochemically reversible ranging from -1.22 to $-1.79 \mathrm{~V}$. This reduction is assigned to a formazanatecentered reduction, and some trends emerge from examining this series. First, we note that complex 2, which has a chelated $\operatorname{Ir}(\mathrm{III})$ center, is significantly more difficult to reduce than any of the $\mathrm{Pt}(\mathrm{II})$ chelates, a trend we previously observed in many mononuclear formazanate complexes. ${ }^{30,31}$ Other insights come from comparing mononuclear $\mathrm{Pt}$ (II) complex 4 to the two dinuclear complexes which contain this building block, $\mathbf{3}$ and $\mathbf{5}$. The reduction potential in 4 is $-1.55 \mathrm{~V}$, which shift anodically to $-1.46 \mathrm{~V}$ when the pyridine is capped with another $\mathrm{Pt}$ (II) unit in 3, and similarly to $-1.48 \mathrm{~V}$ when $\operatorname{Ir}(\mathrm{III})$ binds to the pyridine in $\mathbf{5}$.

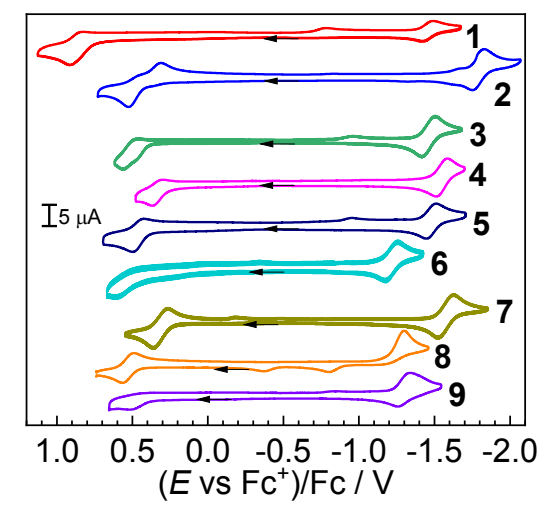

Figure 9. Overlaid cyclic voltammograms of complexes 1-9. CVs were recorded in $\mathrm{CH}_{2} \mathrm{Cl}_{2}$ with $0.1 \mathrm{M} \mathrm{NBu}_{4} \mathrm{PF}_{6}$ supporting electrolyte, using a glassy carbon working electrode and a scan rate of $0.1 \mathrm{~V} / \mathrm{s}$. The arrows indicate the scan direction.

Table 2. Summary of electrochemical data for complexes 1-9.

\begin{tabular}{lcc}
\hline & $\mathrm{E}^{\mathrm{ox}}(\mathrm{V})$ & $\mathrm{E}^{\mathrm{red}}(\mathrm{V})$ \\
\hline $\mathbf{1}$ & 0.88 & -1.45 \\
$\mathbf{2}$ & $0.52^{\mathrm{a}}$ & -1.79 \\
$\mathbf{3}$ & $0.56^{\mathrm{a}}$ & -1.46 \\
$\mathbf{4}$ & 0.34 & -1.55 \\
$\mathbf{5}$ & 0.47 & -1.48 \\
$\mathbf{6}$ & $0.59^{\mathrm{a}}$ & -1.22 \\
$\mathbf{7}$ & 0.31 & -1.58 \\
$\mathbf{8}$ & 0.53 & $-1.30^{\mathrm{a}}$ \\
$\mathbf{9}$ & $0.49^{\mathrm{a}}$ & $-1.33^{\mathrm{a}}$ \\
\hline
\end{tabular}

${ }^{a}$ irreversible waves. $E_{p, a}$ or $E_{p, c}$ is reported.

Likewise, the one-electron oxidation couples are influenced to a small extent, with 3 and $\mathbf{5}$ more difficult to oxidize by $\sim 0.1-0.2$ V. This observation suggests that when the pyridine coordinates to another Lewis-acidic metal center, the formazanate-centered frontier orbitals are stabilized by a small amount, indicating that dinuclear assembly can influence formazanate-based orbital energies. In the Fzb series, we see that the mononuclear precursors $\mathbf{6}$ and $\mathbf{7}$ differ significantly in their reduction potentials. In cationic complex 6 the reduction is anodically shifted to $-1.22 \mathrm{~V}$, compared to $-1.58 \mathrm{~V}$ in neutral cyclometalated complex $\mathbf{7}$, revealing that the secondary ligand, tbbpy or ppy, couples electronically with the formazanate $\pi$ system through the platinum center. Relatedly, cationic complex 6 has a formal oxidation couple that is $\sim 0.3 \mathrm{~V}$ more positive than neutral complex 7. When complex 6 binds $\mathrm{PtCl}_{2}$ and rearranges to complex $\mathbf{8}$, the reduction becomes irreversible and undergoes a slight cathodic shift, whereas the oxidation is minimally perturbed. In the other dinuclear Fzb complex, 9, the reduction is also irreversible, and we propose that it is a concerted two-electron reduction of both formazanates, given the larger observed current compared to the oxidation wave. All that said, in the compounds that have the formazanate core chelated to platinum (3-7 and 9) or bridging two platinum centers (8), the oxidation and reduction potentials are separated by a similar amount, $\sim 1.8 \mathrm{~V}$ in most cases and no larger than $2.0 \mathrm{~V}(3)$, indicating HOMO-LUMO gaps that are all quite similar. Consistent with this, in the UV-vis spectra described above, all of these $\mathrm{Pt}$ (II) complexes have very similar low-energy absorption maxima, ascribed to the formazanate-centered HOMO $\rightarrow$ LUMO $\left(\pi \rightarrow \pi^{*}\right)$ transition.

\section{Conclusions}

In this work, we show that flexidentate, redox-active pyridine-substituted formazanates can template the assembly of a variety of dinuclear assemblies. The formazanates exhibit three distinct binding modes in the five dinuclear complexes, which include diiridium, diplatinum, and mixed platinumiridium constructs. The redox and optical properties are dominated by the formazanate dinucleating ligand, and they do depend on the nuclearity of the complex, the binding mode of the formazanate, as well as the metals that are coordinated. This work shows that these types of formazanates are versatile ligands for the facile assembly of complex structures, where the frontier orbitals of the formazanate can play a large role in determining the electronic structure, redox potentials, and excited-state chemistry of the resulting assemblies. We believe that the two flexidentate formazanates described here, as well as related derivatives, will emerge as ideal scaffolds for the assembly of polynuclear coordination complexes with desirable properties.

\section{Acknowledgements}

We acknowledge the University of Houston and the Welch Foundation (Grant no. E-1887) for funding. 


\section{Notes and references}

1 A. R. Siedle and L. H. Pignolet, Inorg. Chem., 1980, 19, 20522056.

2 B. Budesinsky and J. Svecova, Inorg. Chem., 1971, 10, 313-317.

3 J. B. Gilroy and E. Otten, Chem. Soc. Rev., 2020, 49, 85-113.

4 R. A. Zarkesh, A. S. Ichimura, T. C. Monson, N. C. Tomson and M. R. Anstey, Dalton Trans., 2016, 45, 9962-9969.

5 N. A. Ketterer, H. Fan, K. J. Blackmore, X. Yang, J. W. Ziller, M.-H. Baik and A. F. Heyduk, J. Am. Chem. Soc., 2008, 130, 4364-4374.

6 L. A. Cameron, J. W. Ziller and A. F. Heyduk, Chem. Sci., 2016, 7, 1807-1814.

7 R. R. Maar, A. Rabiee Kenaree, R. Zhang, Y. Tao, B. D. Katzman, V. N. Staroverov, Z. Ding and J. B. Gilroy, Inorg. Chem., 2017, 56, 12436-12447.

8 R. Mondol and E. Otten, Inorg. Chem., 2019, 58, 6344-6355.

9 A. Melenbacher, J. S. Dhindsa, J. B. Gilroy and M. J. Stillman, Angew. Chem. Int. Ed., 2019, 58, 15339-15343.

10 M. Hesari, S. M. Barbon, V. N. Staroverov, Z. Ding and J. B. Gilroy, Chem. Commun., 2015, 51, 3766-3769.

11 S. M. Barbon, V. N. Staroverov and J. B. Gilroy, Angew. Chem. Int. Ed., 2017, 56, 8173-8177.

12 S. M. Barbon, J. V. Buddingh, R. R. Maar and J. B. Gilroy, Inorg. Chem., 2017, 56, 12003-12011.

13 R. R. Maar, R. Zhang, D. G. Stephens, Z. Ding and J. B. Gilroy, Angew. Chem. Int. Ed., 2019, 58, 1052-1056.

14 R. Mondol, D. A. Snoeken, M.-C. Chang and E. Otten, Chem. Commun., 2017, 53, 513-516.

15 J. S. Dhindsa, A. Melenbacher, S. M. Barbon, M. J. Stillman and J. B. Gilroy, Dalton Trans., , DOI:10.1039/C9DT03417J.

16 R. Mondol and E. Otten, Dalton Trans., 2019, 48, 13981-13988.

17 R. Travieso-Puente, J. O. P. Broekman, M.-C. Chang, S. Demeshko, F. Meyer and E. Otten, J. Am. Chem. Soc., 2016, 138, 5503-5506.

18 M.-C. Chang, T. Dann, D. P. Day, M. Lutz, G. G. Wildgoose and E. Otten, Angew. Chem. Int. Ed., 2014, 53, 4118-4122.

19 M.-C. Chang, P. Roewen, R. Travieso-Puente, M. Lutz and E. Otten, Inorg. Chem., 2015, 54, 379-388.

20 D. L. J. Broere, B. Q. Mercado, E. Bill, K. M. Lancaster, S. Sproules and P. L. Holland, Inorg. Chem., 2018, 57, 9580-9591.

21 D. L. J. Broere, B. Q. Mercado, J. T. Lukens, A. C. Vilbert, G. Banerjee, H. M. C. Lant, S. H. Lee, E. Bill, S. Sproules, K. M. Lancaster and P. L. Holland, Chem. - Eur. J., 2018, 24, 94179425.

22 F. Milocco, S. Demeshko, F. Meyer and E. Otten, Dalton Trans., 2018, 47, 8817-8823.

23 F. Milocco, F. de Vries, A. Dall'Anese, V. Rosar, E. Zangrando, E. Otten and B. Milani, Dalton Trans., 2018, 47, 14445-14451.

24 S. Hong, L. M. R. Hill, A. K. Gupta, B. D. Naab, J. B. Gilroy, R. G. Hicks, C. J. Cramer and W. B. Tolman, Inorg. Chem., 2009, 48, 4514-4523.

25 S. Hong, A. K. Gupta and W. B. Tolman, Inorg. Chem., 2009, 48, 6323-6325.

26 N. A. Protasenko, A. I. Poddel'sky, A. S. Bogomyakov, G. K. Fukin and V. K. Cherkasov, Inorg. Chem., 2015, 54, 6078-6080.

27 R. R. Maar, S. M. Barbon, N. Sharma, H. Groom, L. G. Luyt and J. B. Gilroy, Chem. - Eur. J., 2015, 21, 15589-15599.

28 S. M. Barbon, S. Novoa, D. Bender, H. Groom, L. G. Luyt and J. B. Gilroy, Org. Chem. Front., 2017, 4, 178-190.

29 E. Kabir, C.-H. Wu, J. I.-C. Wu and T. S. Teets, Inorg. Chem., 2016, 55, 956-963.

30 E. Kabir, D. Patel, K. Clark and T. S. Teets, Inorg. Chem., 2018, 57, 10906-10917.
31 E. Kabir, G. Mu, D. A. Momtaz, N. A. Bryce and T. S. Teets, Inorg. Chem., 2019, 58, 11672-11683.

32 G. Mu, L. Cong, Z. Wen, J. I.-C. Wu, K. M. Kadish and T. S. Teets, Inorg. Chem., 2018, 57, 9468-9477.

33 G. Mu, Z. Wen, J. I.-C. Wu and T. S. Teets, Dalton Trans., 2020, 49, 3775-3785.

34 R. R. Maar, N. A. Hoffman, V. N. Staroverov and J. B. Gilroy, Chem. - Eur. J., 2019, 25, 11015-11019.

35 N. A. Frolova, S. Z. Vatsadze, A. I. Stash, R. D. Rakhimov and N. V. Zyk, Chem. Heterocycl. Compd., 2006, 42, 1444-1456.

36 N. A. Frolova, S. Z. Vatsadze, V. E. Zavodnik, R. D. Rakhimov and N. V. Zyk, Russ. Chem. Bull., 2006, 55, 1810-1818. 